\title{
Kinetic Roughness of Amorphous Multilayers Studied by Diffuse X-Ray Scattering
}

\author{
T. Salditt, T. H. Metzger, and J. Peisl \\ Sektion Physik der Ludwig-Maximilians-Universität München, Geschwister-Scholl-Platz 1, 80539 München, Germany
}

(Received 28 December 1993)

\begin{abstract}
We apply the scattering geometry of grazing incidence and exit angles to study the diffuse scattering of an amorphous, magnetron sputtered $\mathrm{W} / \mathrm{Si}$ multilayer. Only this technique allows for the full range of parallel momentum transfer necessary to determine the height-height self- and cross-correlation functions from the structure factor of the rough interfaces and the exit-angle-resolved intensity, respectively. The self-correlation functions show the logarithmic scaling behavior predicted by the Edwards-Wilkinson Langevin equation, which describes the kinetic roughening of a growing surface. The cross-correlation functions also agree with those derived from the equation.
\end{abstract}

PACS numbers: $68.55 .-\mathrm{a}, 05.40 .+\mathrm{j}, 61.10 .-\mathrm{i}$

Interface roughness, which inevitably occurs during the nonequilibrium growth of thin layers and layered structures, is currently a topic of intense scientific activity. The surface roughness of a single layer may contain information on the mechanism of growth, its dimensionality and scaling behavior, as shown, for example, by the $\mathrm{x}$-ray reflectivity measurements of $\mathrm{Au}$ on $\mathrm{Si}$ [1].

However, from such specular reflectivity measurements only the average rms roughness can be deduced, whereas lateral interface structures are accessible by measuring the diffuse intensity in nonspecular directions. Let the sample surface be parametrized by a continuous height function $h(x, y)$ and let the plane of reflection be the $x z$ plane. If $\vec{Q}$ denotes the scattering vector, the specular condition is then given by $Q_{x}=Q_{y}=0, Q_{z}>0$, and the diffuse scattering is measured with a parallel momentum transfer $Q_{\|}=\sqrt{Q_{x}^{2}+Q_{y}^{2}}$. Recently, partially self-affine surface structures with isotropic height-height self-correlation functions of the form

$$
C(r):=\left\langle h\left(\vec{r}^{\prime}\right) h\left(\vec{r}^{\prime}+\vec{r}\right)\right\rangle=\sigma^{2} \exp \left[-(r / \xi)^{2 h}\right]
$$

have been reported [2-3], where $\sigma$ is the rms roughness, $h$ is the static or spatial roughening exponent, and $\xi$ is an upper cutoff. This function can be deduced from the structure factor of a rough surface $S_{\text {rough }}(\vec{Q})$, which has been calculated within the distorted-wave Born approximation [4].

In a heterostructure with periodically repeating interfaces between two chemically different materials as in a synthetic multilayer, additional correlations across different interfaces must be expected, the so-called height-height cross-correlations $\left\langle h_{i}\left(\vec{r}^{\prime}\right) h_{j}\left(\vec{r}^{\prime}+\vec{r}\right)\right\rangle$ [5]. If one succeeds in determining these cross-correlations experimentally, access not only to the spatial but also to the temporal aspects of the growth process is obtained, as different interfaces can be attributed to different times $t$ during growth. Under the assumption that the interface structure does not relax after growth, one can thus in principle deduce both scaling exponents that characterize the statistical properties of a growing self-affine surface [6]. However, if the two materials of the heterostructure do not grow symmetrically, separate growth parameters have to be introduced for each type of layer by the same parameters.

We will show that for the $\mathrm{W} / \mathrm{Si}$ multilayer studied here, both the measured self- and the cross-correlations can be understood, if the growth is modeled by the EdwardsWilkinson Langevin equation [7]

$$
\frac{\partial h(\vec{r}, t)}{\partial t}=\nu \Delta h(\vec{r}, t)+\eta(\vec{r}, t) .
$$

where $\nu$ is a coefficient describing how effective the local curvature is in smoothing out the interface profile, while the uncorrelated white shot noise $\eta$ describes the stochastic nature of the deposition process. The analytical solution of this equation in $2+1$ dimensions gives a logarithmic self-correlation function and a "diffusionlike" temporal spread of fluctuations.

Equation (2) was originally proposed to describe a somewhat oversimplified growth model that is hardly expected to be applicable to real systems. However, since then it has been shown in a renormalization approach that any model, assuming the growth velocity along the interface normal to be a function of only the local derivatives of $h(\vec{r}, t)$, obeys the more general Kardar-Parisi-Zhang equation [8]. In some special cases and in early stages of growth, Monte Carlo simulations have shown that the solution of this equation can be approximated by its linearized version Eq. (2) and shows the corresponding scaling exponents before a crossover to the full nonlinear behavior [9].

Multilayers with a nanometer periodicity $d$ in the growth direction are currently developed for various applications that mostly rely crucially on the roughness of the interfaces and on the roughness correlations between them. Recently we have shown that the diffuse scattering of an amorphous $\mathrm{W} / \mathrm{C}$ multilayer around the specular condition at small angles is concentrated in sheets of nonvanishing intensity in reciprocal space at $Q_{z}=n 2 \pi / d[10]$. These so-called diffuse "Bragg sheets" are modulated by standing wave effects whenever the angle of incidence or exit is equal to a Bragg angle, $2 d \sin \alpha=n \lambda$. In the present work, however, these dynamical effects can be neglected, as the data have been recorded at $\alpha_{i}, \alpha_{f} \neq \alpha_{\text {Bragg }}$, and a kinematical approach is thus sufficient. 
We demonstrate in this Letter that the limitations and disadvantages of the conventional diffuse $\mathrm{x}$-ray scattering techniques as described above can be overcome by a scattering geometry normally used for grazing-incidence large angle diffraction (GID), as shown in Fig. 1(a). Here the incident beam strikes the sample surface under an angle $\alpha_{i}$ and is reflected under the angle $\alpha_{f}=\alpha_{i}$. The scattered $\mathrm{x}$ rays are recorded by a position sensitive detector (PSD), which can be set to measure specular or diffuse scattering along $Q_{z}$, by choosing a particular $\alpha_{i}$ and $\alpha_{f}$. As shown in Fig. 1(b), we placed the PSD on the first diffuse Bragg sheet, $Q_{z}=2 \pi / d$. Keeping $\alpha_{i}$ and $\alpha_{f}$ constant and rotating the detector by $2 \theta$ around the normal of the sample surface, we can set $Q_{\|}$to any desired value, without the restriction from which experiments in the plane of reflection suffer. In addition, since $Q_{z}$ is kept always the same, the diffuse intensity does not have to be deconvoluted for the tedious transmission functions ("Yoneda wings") or corrections for effective sample size at small $\alpha_{i}$ and $\alpha_{f}$.

We used two modes of recording the diffuse scattering intensity close to the forward direction; see Fig. 1(b): First, we measure the decay of the integrated intensity

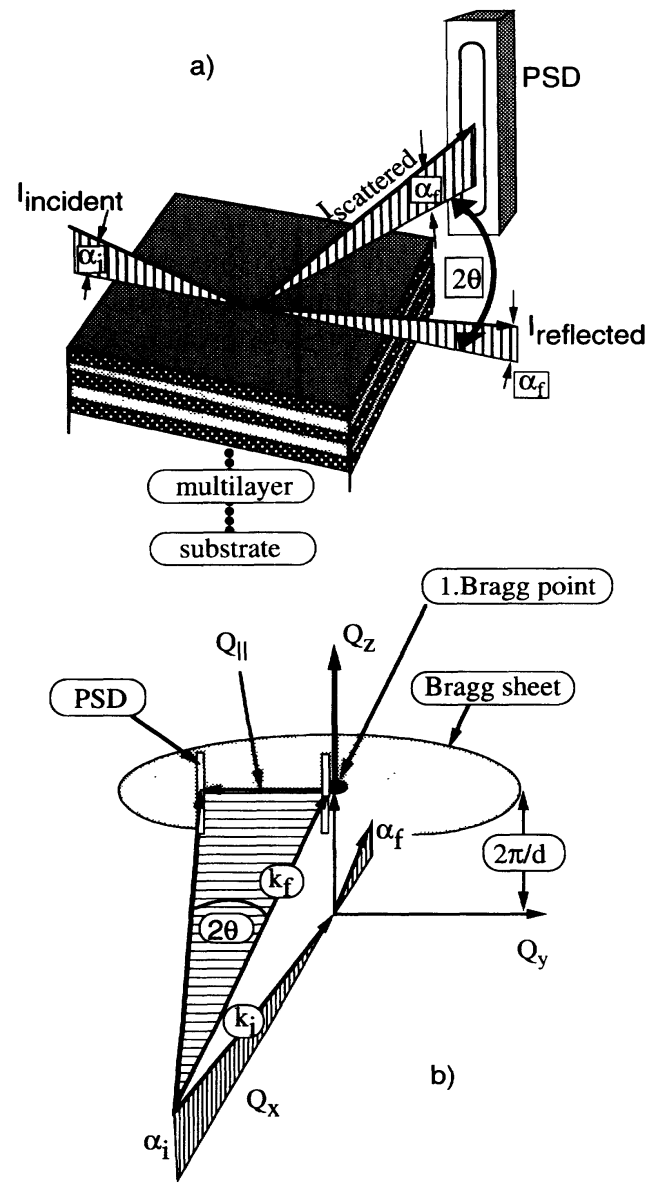

FIG. 1. The scattering geometry at grazing incidence and exit angles $\alpha_{i}, \alpha_{f}$, shown in (a) real and in (b) reciprocal space. over the PSD as a function of $Q_{\|}$. From these data, the self-correlation function averaged over the top 60 interfaces. This is possible, if the intensity is integrated over a range $\Delta Q_{z} \simeq 2 \pi / d$, so that the coherent and incoherent contributions to the diffuse scattering are equally sampled. In the second mode, the spatial resolution of the PSD along $Q_{z}$ was exploited to monitor directly the width of the Bragg sheet as a function of $Q_{\|}$. From the $Q_{z}$ breadth in these scans, the number of interfaces $N$ which contribute coherently to the diffuse scattering is determined as a function of the lateral length scale $r$ of the interface fluctuations. This scattering geometry allows for measurements in a large range of $Q_{\|}$, which is crucial for the determination of the correct correlation function.

For large $Q_{\|}$, this scattering geometry also allows us to investigate the amorphous structure factor $S_{\text {bulk }}(Q)$ of the multilayer at exactly the same scattering depth in which the diffuse scattering of the rough interfaces is measured. We have shown recently how to separate quantitatively the contributions to diffuse scattering resulting from rough interfaces or from amorphous bulk, using their distinctive $Q$ dependences [11].

The experiment was performed at the wiggler beamline $W 1$ of the storage ring Doris III at the HASYLAB/DESY in Hamburg using a wavelength $\lambda=1.44 \AA$. The important beam collimations both in and out of the plane of reflection were set to $\Delta \alpha_{i}=1, \Delta \alpha_{f}=0.1$, and $\Delta 2 \theta=$ $2 \mathrm{mrad}$. The PSD has a sensitive length of $45 \mathrm{~mm}$ and covered a range of $\Delta \alpha_{f}=2.7^{\circ}$ at a distance of $959 \mathrm{~mm}$ behind the sample. As a sample we chose an amorphous $\mathrm{W} / \mathrm{Si}$ multilayer grown by a computer controlled magnetron sputtering machine, using a well polished silicon wafer as a substrate. The multilayer stack consisted of 150 bilayers of periodicity $d=22.8 \AA$. The individual $\mathrm{W}$ and Si layer thicknesses were $d_{\mathrm{W}}=10.8$ and $d_{\mathrm{Si}}=12.0 \AA$, respectively. An average interface rms roughness of $\sigma=$ $3 \AA$ had been determined from reflectivity measurements using $\mathrm{Cu} K_{\alpha 1}$ radiation. The first Bragg sheet of this multilayer is expected to occur at $Q_{z}=2 \pi / d=0.276 \AA^{-1}$. In order to place the center of the PSD on the first diffuse Bragg sheet, we chose $\alpha_{i}=0.98^{\circ}=3.15 \alpha_{c}, \alpha_{c}$ being the critical angle for total reflection at the wavelength used. Since this incidence angle $\alpha_{i}$ is smaller than the first Bragg angle $\alpha_{1 . \mathrm{Bragg}}=1.8^{\circ}, \alpha_{f}$ has to be centered on $2.64^{\circ}$ to meet the condition $Q_{z}=2 \pi / d$. The corresponding scattering depth $\Lambda$ which is a function of $\alpha_{i}$ and $\alpha_{f}$ [12] is then basically absorption controlled. If $\Lambda$ is converted to the number of interfaces $N_{\max }$ that can be investigated, $N_{\max }$ is about 60. This will become important for the evaluation of the width of the Bragg sheets.

Figure 2 shows the $Q_{z}$-integrated intensity of the first Bragg sheet as a function of parallel momentum transfer on a log-log scale. In order to extract the self-correlation function, we need to specify the "window" in $Q_{\|}$, where the measured intensity is dominated by scattering from interface roughness. Towards low $Q_{\|}$this window is limited by the divergence of the primary (and hence the specular) 


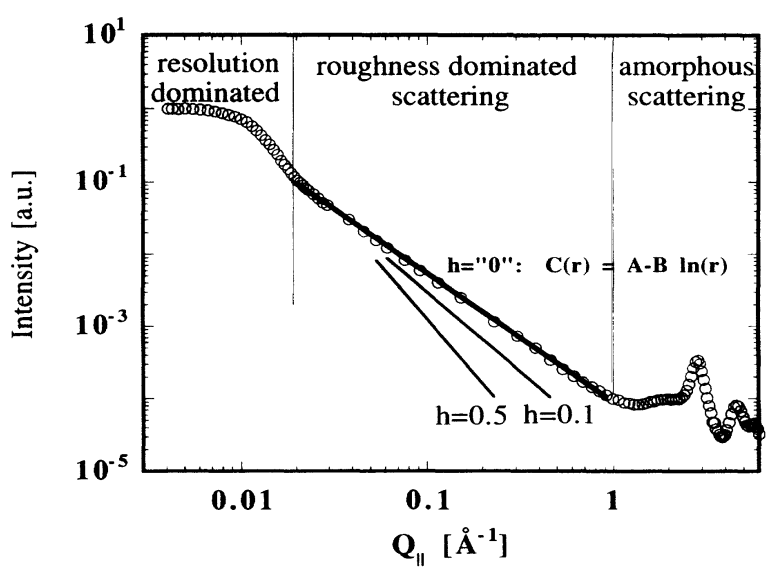

FIG. 2. The decay of the intensity with $Q_{\|}$in the first diffuse Bragg sheet. In the region dominated by scattering from rough interfaces, the fits obtained with the different correlation functions are shown.

beam; towards high $Q_{\|}$it sees the amorphous scattering that is observed in the high angle region $2 \theta \geq 13^{\circ}$ as an essentially incoherent superposition of the $\mathrm{W}$ and $\mathrm{Si}$ amorphous structure factors. In between these limits a comparison of the data with the theoretical predictions made in the framework of the distorted-wave Born approximation [4] can be made. All interfaces are assumed to have the same spatial roughness exponent $h$. The cross-correlations of the interfaces have not been taken into account, as the integration along $Q_{z}$ averages over coherently and incoherently scattered intensity [5]. The structure factor was calculated numerically for the correlation function of Eq. (1) for values of $h=0.1$ and $h=0.3$. At $Q_{\|} \gg 1 / \xi$, only the asymptotic behavior $C(r) \propto r^{2 h}$ of the correlation function is probed, independent of the cutoff length $\xi$ and the special form of the function chosen. Therefore the scattering intensity decays by a power of $\nu$ with $Q_{\|}$. The exponent $\nu$ is a function of $h$ and of $\left(Q_{z} \sigma\right)^{2}$. When the width of the Bragg sheet $\Delta Q_{z}$ is large, the exponent $\nu$ may differ for the two ends of the PSD, and the $Q_{z}$-resolved data have to be taken into account. However, this effect is negligible in our case. A detailed paper on the methological aspects of nonspecular scattering in grazing incidence diffraction geometry and on the data analysis is in preparation. Equation (1) is obviously inconsistent with the data. If, however, a logarithmic roughness characterized by a correlation function of the type $C(r)=A-B \ln r$ is assumed, where $A$ and $B$ are constants, the predicted exponent is $\nu=-2+\left(Q_{z} \sigma\right)^{2} / 2$ [see Eq. (2.38) of Ref. [4]], which is in excellent agreement with our data.

Let us now turn to the $\alpha_{f}$-resolved data, i.e., the intensity distribution along the channels of the PSD for a given value of $2 \theta$. Within the small angle approximation $\sin \alpha_{i} \simeq \alpha_{i}$, the PSD channel number is proportional to $Q_{z}$. In the plane of reflection at $2 \theta=0$, the full width at half maximum (FWHM) of the first Bragg sheet is $2.1 \times 10^{-3} \AA^{-1}$. Increasing $2 \theta$ we observe, along with the strong intensity decay discussed above, a broadening of the Bragg sheet, i.e., an increase of its FWHM, until the modulations along $Q_{z}$ finally vanish for $2 \theta \geq 3^{\circ}$. To indicate the overall behavior, PSD spectra measured in the range $0.1^{\circ} \leq 2 \theta \leq 1^{\circ}$ have been combined in the 3D plot of Fig. 3, showing the intensity as a function of $Q_{z}$ and $Q_{\|}$. The number of coherently interfering layers $N(r)$, defined as the number of interfaces over which the heightheight cross-correlation on a given lateral length scale $r$ is damped to $1 / e$ of its original amplitude, is now calculated from the "thickness" of the sheets

$$
S_{\text {rough }}(\vec{Q}) \sum_{n}^{N}\left|e^{-i n Q_{z} d / 2}\right|^{2} \propto \frac{\sin ^{2}\left(N Q_{z} d / 4\right)}{\sin ^{2}\left(Q_{z} d / 4\right)} .
$$

The values of $N$ are plotted against the corresponding lateral length scale $r=2 \pi / Q_{\|}$in Fig. 4. The saturation of $N(r)$ at a value of about 57 interfaces for $r \geq$ $500 \AA$ is simply due to the fact that absorption limits the number of interfaces probed. The width and the form of the Bragg sheet were studied with and without [Eq. (3)] an attenuation term describing the absorption effect. However, no significant difference in $N(r)$ was found for $r \leq 300 \AA$, so that Eq. (3) can be used for a quantitative analysis in this range.

In order to understand the strong increase of $N(r)$ in the range not affected by absorption, we consider a long wavelength fluctuation in a certain interface developed due to the random nature of the growth process. It is intuitively sensible that this fluctuation will be replicated better across subsequent layers than a small pertubation of a few atomic distances, and we thus expect it not to be damped out within a layer of $d \simeq 20 \AA$. To obtain a quantitative understanding of this phenomenon, we interpret our data along the lines of a multilayer scattering theory by Stearns [13]. In this approach, the frequency

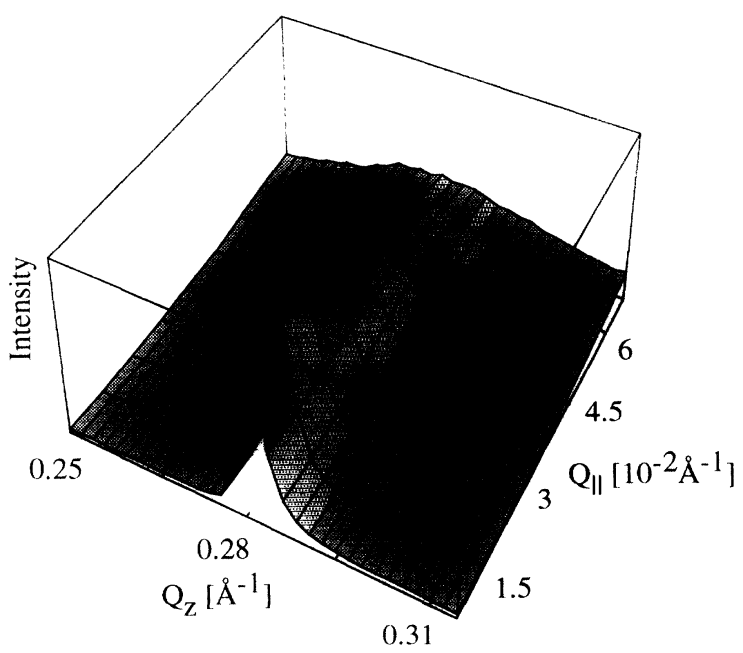

FIG. 3. The intensity distribution of the Bragg sheet as a function of $Q_{z}$ and $Q_{\|}$. The peaks of nine different PSD curves recorded at $2 \theta=0.1^{\circ}, 0.2^{\circ}, \ldots, 1^{\circ}$ have been combined, with logarithmic scaling of the peak heights. The increase in the FWHM with $Q_{\|}$indicates the decay of height-height crosscorrelations for the shorter wavelength components. 


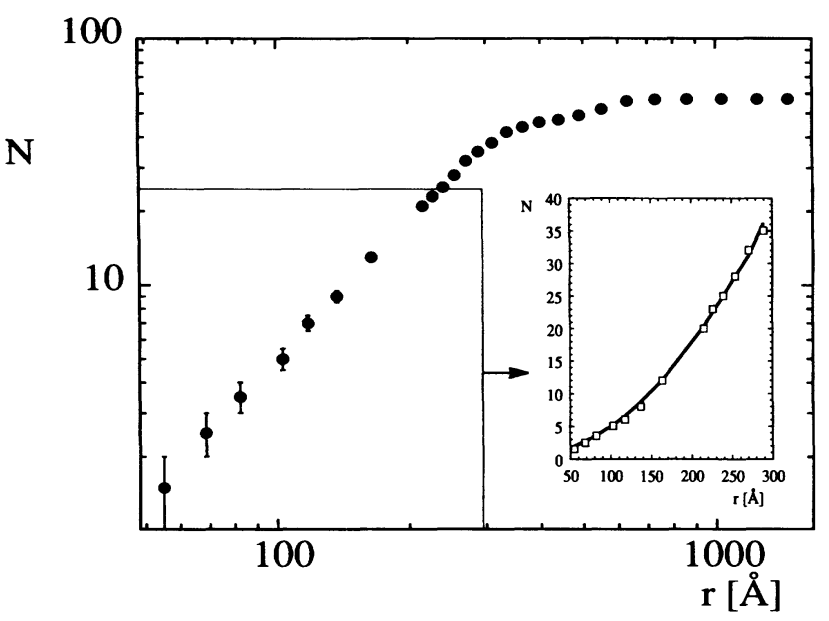

FIG. 4. The number of correlated interfaces with $N$ as a function of the lateral length scale $r$. For $r \geq 500 \AA$ absorption limits, shown is the maximum number of interfaces that add up coherently. In the range $50 \AA \leq r \leq 300 \AA$, the theory of Stearns $[13,14]$ is used to fit the data, as shown in the inset.

spectrum of the $i$ th interface $w_{i}(f)$ consists of an intrinsic roughness $h_{i}(f)$, induced by the growth of that particular layer itself, and of a replication term $a_{i}(f)$ that describes the coupling to the roughness of the underlying layer,

$$
w_{i}(\vec{f})=h_{i}(\vec{f})+a_{i}(\vec{f}) w_{i-1}(\vec{f}) .
$$

The intrinsic frequency spectrum is the Fourier transform of a single layer height $h(\vec{r}, t)$ of average thickness $t$ grown on a perfectly flat substrate,

$$
h_{i}(\vec{f})=\frac{1}{A} \int_{A} h(\vec{r}, t) \exp [2 \pi \vec{f} \cdot \vec{r}] d \vec{r} .
$$

So far, the model is still quite general. One can now make the specific assumption that the replication factor is diffusionlike in agreement with the solution of Eq. (2), e.g., given by

$$
a_{i}(\vec{f})=\exp \left[-4 \pi^{2} \nu_{i} d_{i} f^{2}\right]
$$

where $\nu_{i}$ and $d_{i}$ are the relaxation parameter and the thicknesses of the W and Si layers, respectively.

It has further been shown [14] that, with the assumption of Eq. (4) and Eq. (6), the number $N(r)$ of correlated interfaces is given by

$$
N(r)=\frac{2}{\ln \left\{\left[1+(2 \pi)^{4} d_{\mathrm{W}} \nu_{\mathrm{W}} / r^{2}\right]\left[1+(2 \pi)^{4} d_{\mathrm{Si}} \nu_{\mathrm{Si}} / r^{2}\right]\right\}},
$$

where $d_{\mathrm{W}}$ and $d_{\mathrm{Si}}$ are the layer thicknesses of the two components. We now use this formula to make a least squares fit to the data for $r \leq 300 \AA$, i.e., in the region not limited by absorption (see the inset of Fig. 4). The best fit was obtained for parameters $\nu_{\mathrm{Si}}=0.28 \pm 0.04 \AA$ and $\nu_{\mathrm{W}}=0 \pm 0.04 \AA$. This result indicates the two interfaces $\mathrm{W} / \mathrm{Si}$ and $\mathrm{Si} / \mathrm{W}$ are not symmetrically rough and that one component is efficient in smoothing out fluctuations, while the other does not contribute to the smoothening.
However, as $\nu_{\mathrm{W}}$ and $\nu_{\mathrm{Si}}$ enter symmetrically in Eq. (7), it is not possible to decide which of the two interface types is the rough one and which is the smooth one. This problem could be solved in a future investigation from the comparison of single $\mathrm{Si}$ and $\mathrm{W}$ layers grown under the same conditions or from transmission electron micrographs of the multilayer sample. For the present work, it seems more appropriate to define an average parameter that describes the growth after coarse graining over the individual Si and W layers. In this case, Eq. (7) is replaced by

$$
N(r)=\frac{2}{\ln \left[(1+2 \pi)^{4} d \nu / r^{2}\right]},
$$

and the fit in Fig. 4 gives a value of $\nu=0.13 \pm 0.02 \AA$ that now describes the average relaxation parameter of one bilayer.

To conclude, we have measured the height-height selfand cross-correlations in an amorphous $\mathrm{W} / \mathrm{Si}$ multilayer. In contrast to conventional methods used for this task, we chose the scattering geometry typically used for large angle diffraction at grazing incidence and exit angles, allowing for a maximum range of parallel momentum transfer. The results have been interpreted along the lines of kinetic roughening theory. Temporal aspects of roughening are accessible from the multilayer sample, as different interfaces correspond to different times of growth. Good agreement of the data with the predictions of the Edwards-Wilkinson equation (2) was found both for the height-height self- and cross-correlation functions.

This work was supported by the Bundesministerium für Forschung und Technologie under Contract No. 055WMAXI5. We thank S. C. Moss and H. Spohn for fruitful discussions, the HASYLAB for its hospitality, and $\mathrm{H}$. Rhan for technical assistance.

[1] H. You et al., Phys. Rev. Lett. 70, 2900 (1993).

[2] W. Weber and B. Lengeler, Phys. Rev. B 46, 7953 (1992).

[3] D. E. Savage et al., J. Appl. Phys. 69, 1411 (1991).

[4] S. K. Sinha et al., Phys. Rev. B 38, 2297 (1988).

[5] Y. H. Phang et al., J. Appl. Phys. 74, 3181 (1993).

[6] For an overview, see J. Krug and H. Spohn, in Solids Far from Equilibrium, edited by C. Godreche (Cambridge Univ. Press, New York, 1992).

[7] S. F. Edwards and D. R. Wilkinson, Proc. R. Soc. London A 381, 17 (1982).

[8] M. Kardar, G. Parisi, and Y.C. Zhang, Phys. Rev. Lett. 56, 869 (1986).

[9] J. Villain, J. Phys. I (France) 1, 19 (1991).

[10] X. Jiang, T. H. Metzger, and J. Peisl, Appl. Phys. Lett. 61, 8 (1992).

[11] T. Salditt $e$ a al., in Proceedings of the Europhysics Industrial Workshop-9, Eindhoven, 1993 (to be published).

[12] H. Dosch, Critical Phenomena at Surfaces and Interfaces, Springer Tracts in Modern Physics Vol. 126 (SpringerVerlag, Berlin, 1992), p. 8.

[13] D. G. Stearns, J. Appl. Phys. 71, 4286 (1992).

[14] E. Spiller, D. Stearns, and M. Krumrey, J. Appl. Phys. 74, 107 (1993); Appl. Opt. 32, 6969 (1993). 


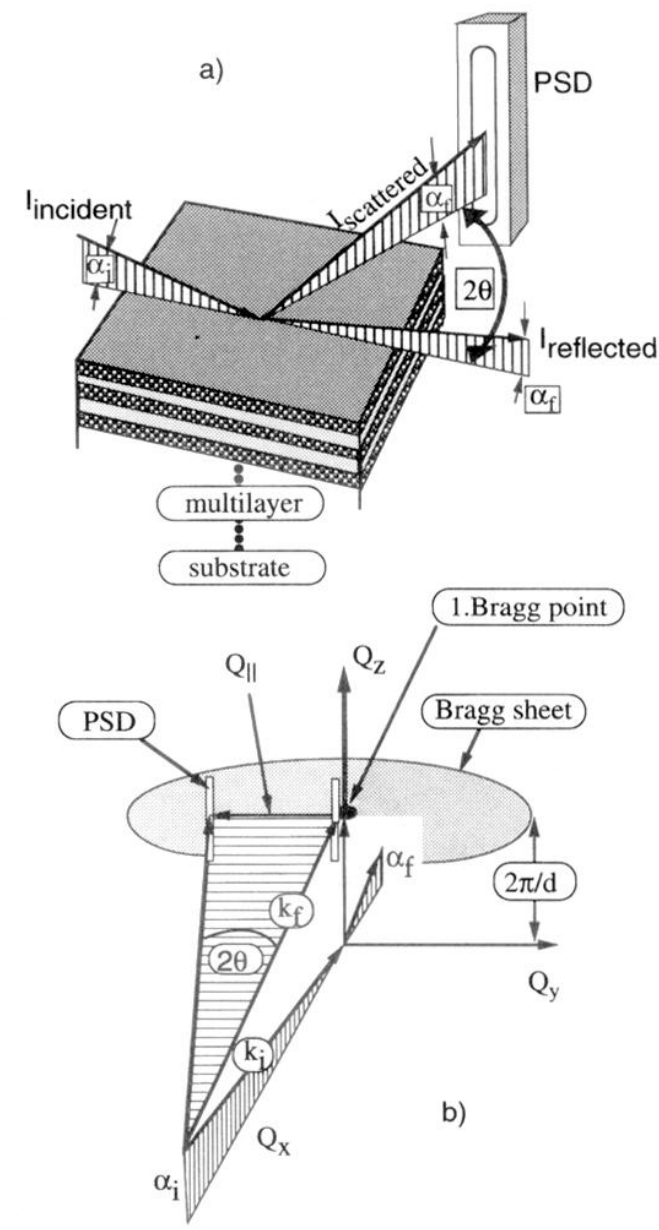

FIG. 1. The scattering geometry at grazing incidence and exit angles $\alpha_{i}, \alpha_{f}$, shown in (a) real and in (b) reciprocal space. 


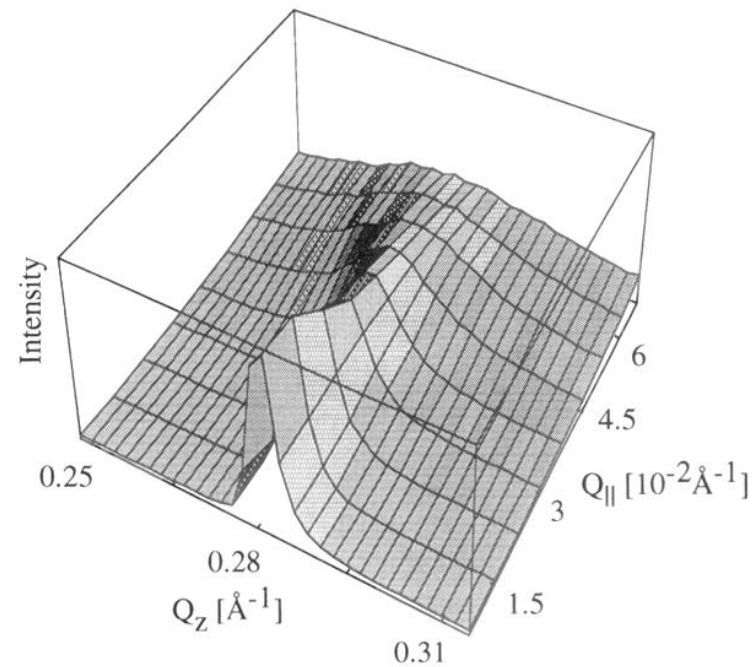

FIG. 3. The intensity distribution of the Bragg sheet as a function of $Q_{z}$ and $Q_{\|}$. The peaks of nine different PSD curves recorded at $2 \theta=0.1^{\circ}, 0.2^{\circ}, \ldots, 1^{\circ}$ have been combined, with logarithmic scaling of the peak heights. The increase in the FWHM with $Q_{\|}$indicates the decay of height-height crosscorrelations for the shorter wavelength components. 